\title{
Ontology-based Context Modeling to Facilitate Reasoning in a Context-Aware System: A Case Study for the Smart Home
}

\author{
M. Robiul Hoque, M. Humayun Kabir, Keshav Thapa and Sung-Hyun Yang \\ Department of Electronic Engineering, \\ Kwangwoon University, 139-701 Seoul, Republic of Korea \\ E-mail: \{robiul, humayun, kshavthapa, shyang\}@kw.ac.kr
}

\begin{abstract}
A context-aware system provides services based on the current context of the environment and user activities. So, context management and reasoning in context-aware systems are important tasks. A formal context model based on ontology can play a vital role in facilitating reasoning by formally representing domain knowledge. This paper presents an ontology-based reusable generic context model for context-aware systems. This model provides a context vocabulary and structure for contexts and their semantics which are essential for reasoning. We evaluate the effectiveness of this model for both ontology and rule-based reasoning in the smart home domain and the result we obtain is promising.
\end{abstract}

Keywords: Smart home, Context modeling, Ontology, FOPL, Reasoning

\section{Introduction}

A context is any information that can be used to characterize the situation of entities that are considered relevant to the interaction between a user and an application, including the user and the application themselves [1]. To make high-level contexts semantically, context reasoning is required. Context reasoning means to automatically deduce further implicit facts from previously given explicit context information. In fact, ontology-based context modeling could help reasoning using logic inferring rules.

Context modeling is the specification of all entities and the relations between these entities that are needed to describe the context as a whole. In this paper, we adopt ontology-based context modeling. Where ontology [2] is a formal explicit definition of a shared conceptualization and has many benefits such as knowledge sharing, logic inference, and knowledge reuse. The main benefit of this model is that it enables a formal analysis of domain knowledge such as performing context reasoning using first order predicate calculus.

There are many ontology languages such as RDF(S), OIL, DAML+OIL and OWL. OWL [3] is divided into three languages- OWL Lite, OWL DL, and OWL full. We adopt OWLDL to represent context ontology which is sufficient to describe the necessary context.

Some researchers have constructed ontologies for contexts in a specific domain to reach the goals of knowledge sharing across distributed systems. In Ref. [4] authors introduced extensible context ontology (CONON) for pervasive computing environments. CONON defines four basic concepts of Activity, Location, Person, and ComEntity. The environment and time concepts are not defined. COBRA, proposed by Chen et al. [5], uses ontology to describe person, places, and intentions. COBRA does not have any general or upper level ontology, so it would be difficult to ensure knowledge reuse and knowledge sharing. Most of the researchers represent ontology-based context modeling for inferring personalized service in pervasive environment but these models are not adjustable for extended domains. Obviously, our proposed two-leveled ontology model 
provides a reusable generic structure and facilitates context reasoning. Our case study shows that this model is sufficiently expressive to satisfy required queries that relate to smart home services.

The rest of this paper is organized as follows. In Section 2, ontology-based context model for the smart home domain is presented in details. In section 3, context managements and reasoning methods are discussed and finally in section 4 , we conclude this paper and indicate future plan.

\section{Context Model for the Smart Home Domain}

We propose a two-leveled ontology model for contextual information: an upper level and lower level ontology. The upper ontology provides definitions for general-purpose terms and acts as a foundation for specific domain ontology. The lower ontology, on the other hand, provides domain specific knowledge to context-aware applications as Figure 1 shows.

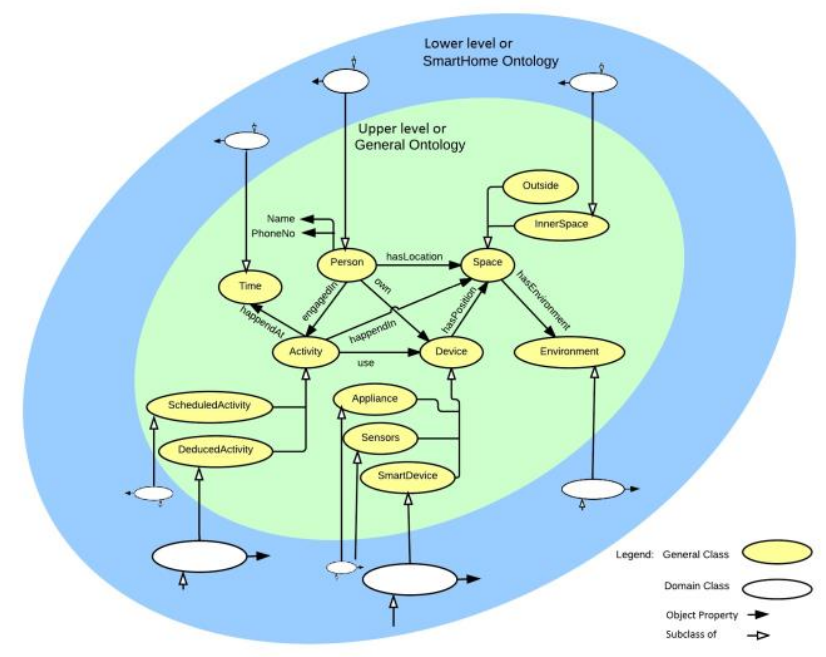

\section{Figure 1. Ontology Levels Where Concepts In The Inner Part Represent Upper Level Or General Ontology, And Concepts In The Outer Part Represent Lower Level Or Domain Specific Ontology}

In our model, we categorize context entities mainly into person, device, space, environment, and time classes which establish the upper ontology. This classification is important in our context modeling process where context representation depends on these entities and the relationships created between them. Although we can easily reuse this model in different domains such as office, classroom, or car, for simplicity we will describe this model by taking the smart domain as an example. However, each class may be divided into several sub-classes. For example, space emphasizes the spatial aspects of a domain. Space has two subclasses, Inner space and outside. Again, the inner spaces of our smart home are divided into room, kitchen, and entry, and represent a lower ontology for the home domain. Furthermore, room is divided into bedroom, living room, bathroom, and storage room. We define the necessary object properties such as hasLocation, hasEnvironment and so on which present the relationships between classes. We define some inverse properties e.g., isLocationOf is an inverse property of hasLocation. Figures 2 and 3 shows a partial ontology of our context model written under the Protégé editor and owl syntax definition of the domain context respectively 


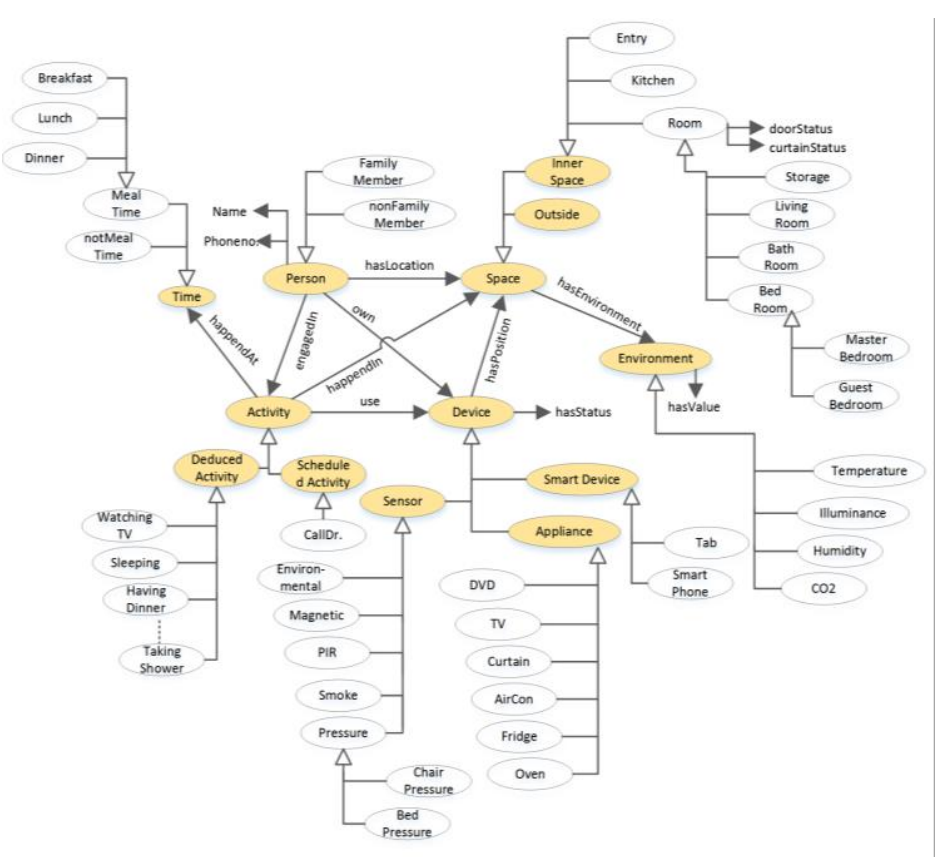

\section{Figure 2. A Part Of The Domain Ontology Where Brown Ellipses Represent General Ontology And White Ellipses Represent Smart Home Domain Ontology}

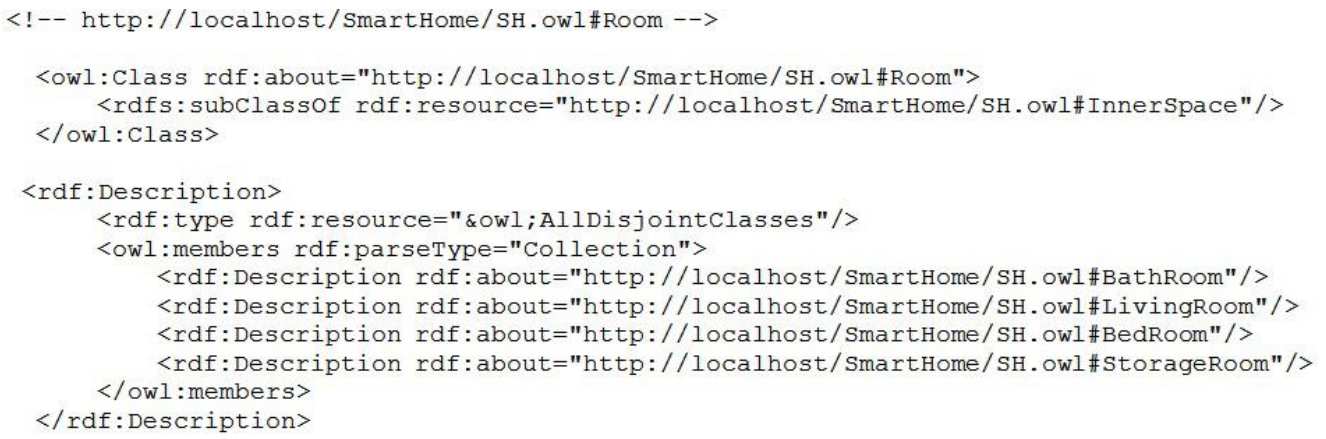

Figure 3. Shows A Part Of The Context Definition Using Owl Syntax

Our context model is semantically rich enough to properly describe concepts, concept hierarchies and their relationships. So, this model can help to expand further knowledge from existing knowledge. For example, Figure 4 illustrates a detected person, Mr. Hong, who is a FamilyMember, his location is MasterBed where BedPressure sensor is $\mathrm{ON}$ and there is no motion. Using this information, a reasoner can deduce that Mr. Hong's current activity is sleeping, as Eq. (1) shows.

In our model, we represent contexts based on first-order predicate calculus where predicate and value represent a context of the subject with respect to a specific property. The basic model has the form predicate(subject, value) or predicate(subject), in which the subject is a set of subject names (e.g., a person, space, or device), predicate is a set of predicate names (e.g., haslocation, hasStatus) and value is the set of all values of subjects (e.g., living room, on, false). For example, haslocation(MrHong, bathroom) means that Mr. Hong is located in the bathroom. 


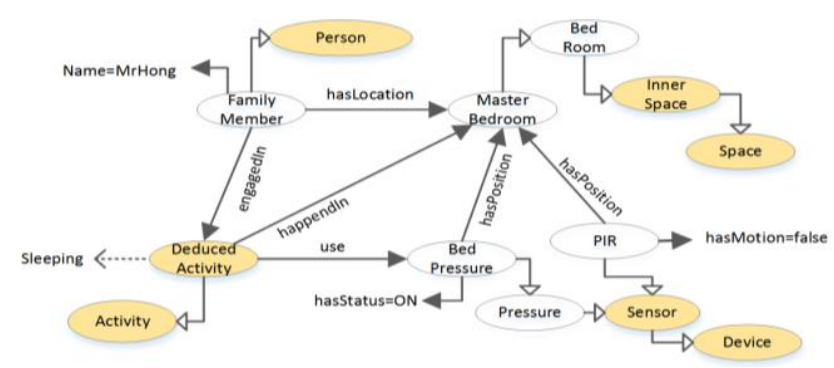

Figure 4. A Part Of Ontology That Shows The Deduced Activity Is Sleeping

\section{Context Reasoning}

Reasoning is a process to reason about various types of contexts and their properties. For our developed middleware [6], we use this context model where two types of reasoning methods are adopted: ontology reasoning, and user-defined rule-based reasoning. Figure 5 shows an overview of our context processing model.

\subsection{Ontology Reasoning}

The ontology reasoning is responsible for checking class consistency and the implied relationships among entities. Ontology reasoning is useful to compute subsumption. Moreover, our smart home implementation uses ontology as a provider of domain context vocabularies and implies relationships between concepts.

\subsection{Rule-based reasoning}

The need for reasoning arises because not all contexts can be gathered from sensed information. For rule-based reasoning, we define a set of rules to assert high-level context from low-level context. Equation (1) shows a rule to deduce a person's current status. This reasoning is provided by the specially designed Reasoning Engine, shown in Figure 5 , using forward chaining algorithm.

Of

Person $(? x) \wedge \operatorname{BedRoom}(? y) \wedge P I R(? r) \wedge$ BedPressure(?p)$\wedge$ hasLocation $(? x, ? y) \wedge$ isPosition

$(? y, ? r) \wedge$ isPosition $O f(? y, ? p) \wedge$ hasMotion $(? r$, false $) \wedge$ hasStatus $(? p, O N) \rightarrow$ engagedIn(?x, Slee ping)

Although our experimental result shows that the reasoning delay increases when the no. of contexts or rule set increases, by analyzing the domain contexts and rule set, we can conclude that this model is quite promising in the smart home domain for non-time-critical applications.

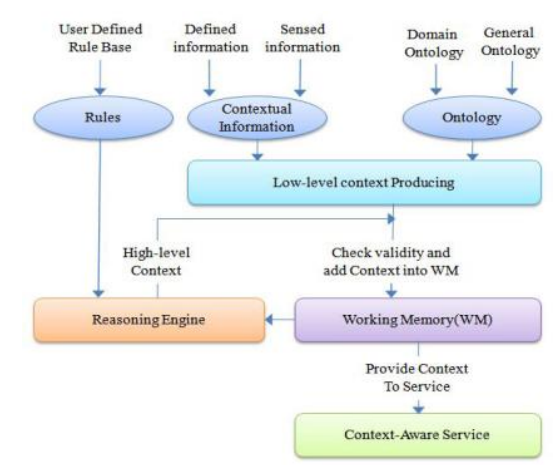

Figure 5. A Context Management And Reasoning Model 


\section{Conclusions}

In this research work, we represent a generic context model based on ontology and reasoning technique with implementation in the smart home domain. This model is sufficiently expressive to satisfy required queries about the smart home context. However, there is scope to improve this model through integrating statistical method for uncertain context reasoning.

\section{Acknowledgements}

This work was supported by the Industrial Strategic Technology Development Program (10041788, Development of Smart Home Service based on Advanced ContextAwareness), funded by the Ministry of Trade, Industry \& Energy (MI, Korea). The present research has been conducted by Research Grant of Kwangwoon University in 2015.

\section{References}

[1] A. K. Dey and G. D. Abowd, "Towards a better understanding of context and context-awareness", Proceedings of Workshop on the what, who, where, when and how of context-awareness, (2000).

[2] D. Fensel, "Ontologies, A silver bullet for Knowledge Management and Electronic Commerce", ISBN 3540-41602-1, (2000).

[3] "W3C, OWL Web Ontology Language Overview, Recommendation", http://www.w3.org/TR/2004/REC-owl-features-20040210/, (2005).

[4] X. Wang and et al., "Ontology based context modeling and reasoning using owl", Proceedings of Workshop on Context Modelling and Reasoning at IEEE Int'l Conference on Pervasive Computing and Communication, (2004), USA.

[5] H. Chen, T. Finin and A. Joshi, "An ontology for context-aware pervasive computing environments", Special Issue on Ontologies for Distributed Systems, knowledge Engineering Review, (2003).

[6] M. R. Hoque, M. H. Kabir, G. Marreiros and S. -H. Yang, "Cooperative Context Dissemination in Smart Home System, a Middleware Based Approach", Proceedings of the 1st International Joint Conference on Convergence (IJCC 2015), (2015) February, Vietnam.

Authors

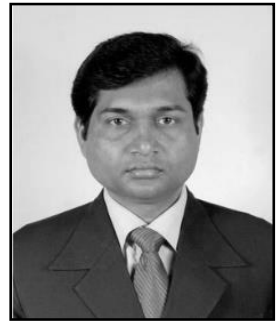

M. Robiul Hoque, He has received B.Sc (Hon's) and M.Sc. degree in Computer Science and d engineering from Islamic University, Kushtia, Bangladesh, in 2003 and 2004 respectively. $\mathrm{He}$ is an Assistant Professor at Islamic University, Kushtia, Bangladesh. Now, he is pursuing his Doctoral degree at Kwangwoon University, Republic of Korea. His main research interests include Context-Aware System, Ubiquitous Computing, and Smart Home, Sensor Networks, Image and Speech processing.

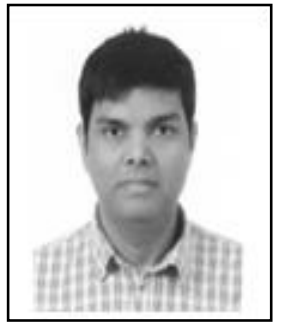

M. Humayun Kabir, He has received his B.Sc (Hon's) and M.Sc degree in Applied Physics, Electronics and Communication Engineering from Islamic University, Kushtia, Bangladesh, in 2001 and 2003 respectively. He is an Assistant Professor at Islamic University, Kushtia Bangladesh. Now, he is pursuing his Doctoral degree at Kwangwoon University, Seoul, Republic of Korea. His main research interests are Context-Aware Systems for the Smart Home, Embedded Systems, M2M, Sensor Networks, Image and Speech processing. 

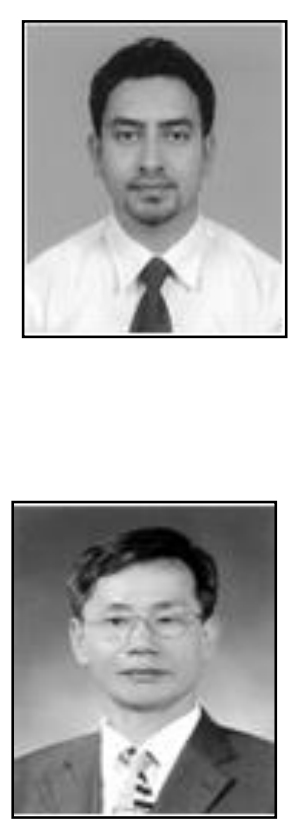

Sung-Hyun Yang, He has received his B.S. and M.S. degree in Electrical Engineering from Kwangwoon University, Seoul, Republic of Korea, in 1983 and 1987 respectively. He completed his Ph.D. from Kwangwoon University in 1993. He is a Professor in Electronic Engineering at Kwangwoon University, Seoul, and Republic of Korea. He is a Director of the Ubiquitous Home Network Center, Kwangwoon University. He was a Research Scientist at Boston University from 1996 to 1998. He was Chairman of the Home Network Market Activation Section, Korean Association for Smart Home from 2007 to 2008. His main research interests are Digital Logic, Embedded Systems, M2M, Next Generation Ubiquitous Home Networks, and Context-Aware System. 